\title{
Nanosecond Laser Shock Microforming of Thin Metal Components
}

\author{
José Luis OCAÑA, Miguel MORALES, Juan Antonio PORRO, Oscar GARCÍA, \\ Juan José GARCÍA-BALLESTEROS and Carlos MOLPECERES \\ Centro Láser UPM. Universidad Politécnica de Madrid \\ Campus Sur UPM. Edificio La Arboleda Ctra. de Valencia, km. 7,300. \\ 28031 Madrid. SPAIN \\ E-mail: jlocana@etsii.upm.es
}

\begin{abstract}
Laser shock microforming is conceived as a non-thermal laser forming method based on the high intensity laser induced shock waves capability to modify the tensional state of thin targets. The technique has the advantages of laser thermal forming (non-contact, tool-free and high precision), but, additionally, its minimally thermal character allows the preservation and improvement of mechanical material properties by inducing appropriate residual stress fields. In particular, the induction of compressive residual stress fields on the target surface is a desirable feature introducing additional protection of the formed parts against corrosion and fatigue crack propagation.
\end{abstract}

The use of ns laser pulses provides a suitable parameter matching for the laser forming of an important range of sheet components used in MEMS that, preserving the short interaction time scale required for the predominantly mechanic (shock) induction of deformation residual stresses, allows for the successful processing of components in a medium range of miniaturization particularly important according to its frequent use in such systems.

In the present paper, a discussion is presented on the physics of laser shock microforming and the influence of the different effects on the net bending angle. The experimental setup used for the experiments, the sample fabrication procedure and experimental results on the influence of repeated laser pulses on the net bending angle are also presented.

DOI: 10.2961/jlmn.2009.01.0011

Keywords: Laser micro-scale shock forming, forming mechanism, numerical modeling, shock wave, micromanufacturing

\section{Introduction}

The increasing demands in MEMS fabrication are leading to new requirements in production technology [1-3]. Especially the packaging and assembly require high accuracy in positioning and high reproducibility in combination with low production costs. Conventional assembly technology and mechanical adjustment methods are time consuming and expensive.

Accurate positioning of smallest components represents an up-to-date key assignment in micro-manufacturing. It has proven to be more time and cost efficient to initially assemble the components with widened tolerances before precisely micro-adjusting them in a second step [2].

Provided that as mounted micro components are typically difficult to access and highly sensitive to mechanical forces and impacts, contact-free laser adjustment processes offer a great potential for accurate manipulation of micro devices.

Laser shock forming is conceived as a non-thermal laser forming method using the shock wave induced by laser irradiation to modify the curvature of the target $[4,5]$. It has the advantages of laser thermal forming (non-contact, tool-free and high efficiency and precision) [1, 2] and, additionally, its non-thermal process makes it possible to maintain material properties or even improve them by inducing compressive stress over the target surface, a desirable since it is important in industry for shaped metal parts to resist cracks from corrosion and fatigue.
In this paper, laser shock micro-forming is studied using both numerical and experimental methods for a thin metallic film in a one-side pinned configuration (see figure 1). The physics of laser shock microforming and the influence of the different effects on the net bending angle are studied.

The experimental setup used for the experiments, the sample fabrication process and experimental results of influence of number of laser pulses on the net bending angle are also presented.

The effect of the laser spot position on the deformation mechanism is investigated experimentally and data obtained from experiments are then used to validate the corresponding simulation model.

The sample curvatures before and after laser microscale shock forming were measured using confocal microscopy in order to find the net bending effect of the process.

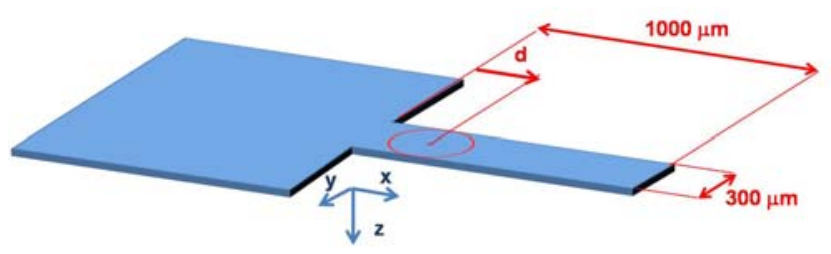

Fig. 1. Schematic diagram of thin film beam and definition of distance d (distance to thin film/beam union). 


\section{Physics of Laser Shock Microforming}

Laser Shock Microforming $(\mathrm{LS} \mu \mathrm{F})$ is based on the application of a high intensity pulsed laser beam (I $>10^{9}$ $\mathrm{W} / \mathrm{cm}^{2} ; \tau<50 \mathrm{~ns}$ ) on a metallic target forcing a sudden vaporization of its surface into a high temperature and density plasma that immediately develops inducing a shock wave propagating into the material. When the laser is switched off, the plasma continues to maintain a pressure which decreases during its expansion.

In Laser Shock processes the material is stressed and deformed in a dynamic way, with strain rates exceeding $10^{6} \mathrm{~s}^{-1}$. The actual material yield strength is taken according to Von Mises' criterion.

Considering a reference thin metal stripe of the type shown in figure 1 plastic deformation induced by the shock wave generates a residual stress distribution. Stress in the direction of the metal stripe $\left(\sigma_{\mathrm{xx}}\right)$ in points close to the incidence surface results to be compressive whereas in the rear surface the residual stresses field is predominantly tensile (see figure 2). This stress distribution produces a local bending $\left(\beta_{\mathrm{s}}\right)$ in the direction of the laser beam (see figure 2).

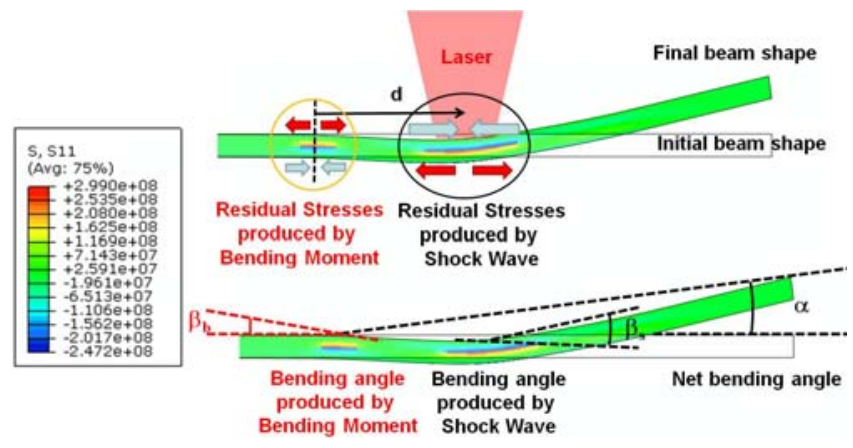

Fig. 2. Schematic diagram of thin film deformation and stresses and definition of bending angles

The pressure is applied by the laser incidence at some distance, $d$, from the clamping (or bulk material) and this generates a bending moment which depends on the laser spot position and energy. Additionally bending moment can produce plastic deformation (near the clamping). In this case stress in the direction of the metal stripe $\left(\sigma_{\mathrm{xx}}\right)$ in points close to the incidence surface is predominantly tensile whereas in the rear surface it is predominantly compressive. This stress distribution produces a local bending $\left(\beta_{\mathrm{b}}\right)$ in the opposite direction of the laser beam (see again figure 2).

The combination of this two effects produce the net bending angle $(\alpha)$, determined for small angles as the ratio between the apparent bar end displacement to the bar length (see figure 2).

This net bending angle can be optimized as a function of the laser characteristics, namely pulse duration, wavelength and energy and other process parameters, namely the laser spot radius, spot position, geometry and the opti$\mathrm{cal}$, thermal and mechanical properties.

When the bending angle produced by bending moment is dominant, the bulk material deformation has a deleterious effect on net bending angle.

\section{Model description}

The developed calculational model is integrated by two principal modules conceived respectively for the analysis of the problem of laser shock waves generation and propagation under two different but complementary approaches [5-7].

On one side, LSPSIM is a one-dimensional model intended for the estimation of the pressure wave applied to the target material in Laser Shock experiments [6,7].

LSPSIM describes the material-tamper gap assuming an only phase of evolution that can be extended to the end of the processes, i.e., obtains the target-confining medium gap amplitude by solving the coupled system of energy and impulse equations subject to the thermofluiddynamic conditions imposed by the laser energy deposition.

On the other hand, on the basis of the time-dependent pressure profile calculated by LSPSIM, the HARDSHOCK code (based in the FEM commercial code ABAQUS ${ }^{\circledR}$ ) solves the shock propagation problem into the solid material, with specific consideration of the material response to thermal and mechanical alterations induced by the propagating wave itself (i.e. effects as elastic-plastic behaviour, changes in elastic constants, phase changes, etc.).

For the kind of problems considered in this paper, a 3D version of the HARDSHOCK code is used. From the point of view of time differencing, the usual strategy of explicit differencing for the initial fast shock propagation phase followed by standard implicit differencing for the analysis of the final residual stresses equilibrium is used. The onset of the implicit phase is defined to apply once all plastic deformation has taken place.

Concerning material behaviour a dynamic elastic limit has to be used. In the reported calculations such limit has been fixed according to Johnson-Cook model [8], applicable to the typical behaviour shown by the different materials through their dynamic strain-stress curve. The actual material yield strength is taken according to Von Mises' criterion.

A sample of the 3D simulated geometry is shown in figure 3. It corresponds to the incidence of a uniform laser beam of cylindrical shape on a planar surface with a pinned end as boundary condition. The FEM element used for the mechanical simulation is an 8-node brick reduced integration with hourglass control, namely C3D8R.

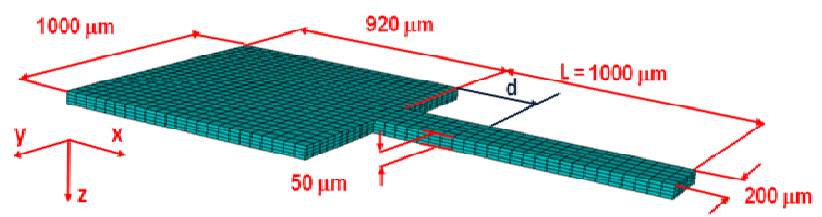

Fig. 3. Geometrical properties of a typical FEM Model used for the numerical simulation of one-end pinned thin metal strip subject to laser shock microforming.

\section{Numerical results}

The described model has been applied to study the mechanical effect of laser pulse energy and laser spot position on the net metal sheets bending angle under the reasonable hypothesis of weak thermal influence (see reference 9). All 
the results presented in this paper refer to Stainless Steel AISI 304 and samples of $50 \mu \mathrm{m}$ thickness and $200 \mu \mathrm{m}$ width irradiated with a 10 ns pulsed Nd:YAG laser operating at $1064 \mathrm{~nm}$ and with a spot diameter of $175 \mu \mathrm{m}$.

\subsection{Analysis of the influence of laser pulse energy on the net bending angle}

As a first step in the analysis, the influence of the laser pulse total energy on the bending angle of typical specimens has been analyzed. In figure 4 the thin sheet deformations for different laser pulse energies are displayed. One only laser pulse incident on a selected area of the specimen (laser spot centered at $d=L / 3$ ) has been considered for this simulation.

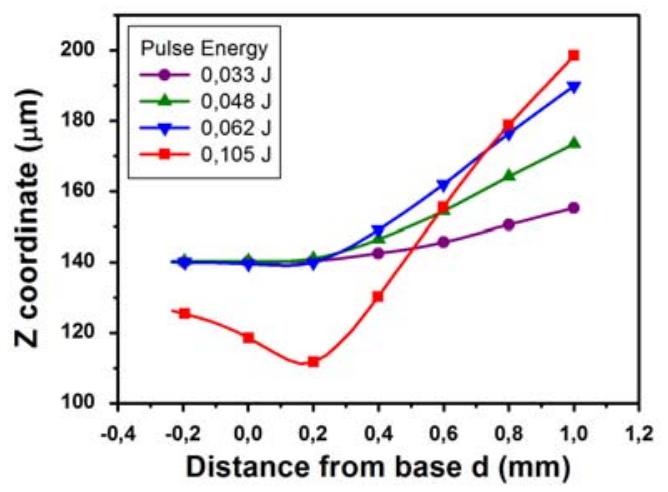

Fig. 4. Numerical deformation curves of reference thin sheet specimens subject to ns laser pulses irradiation at $d=L / 3$ for different laser pulse energies.

The study of net bending angle of the metal sheet as a function of the laser energy pulse (see figure 5) shows that, after a critical value of pulse energy, the bending angle $\left(\beta_{\mathrm{b}}\right)$ produced by the bending moment starts to have a deleterious effect on the net bending angle $(\alpha)$.

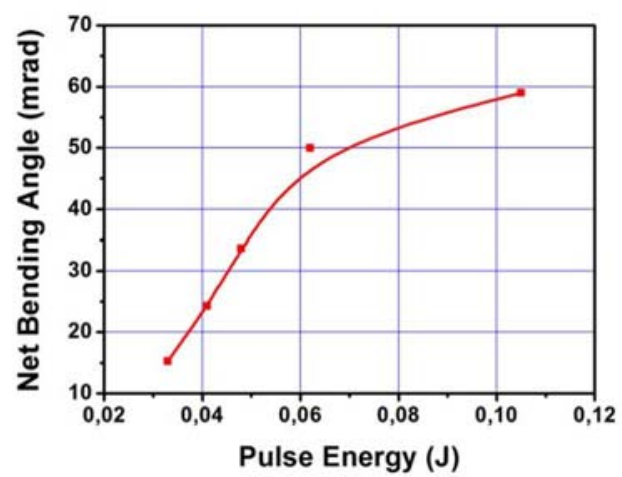

Fig. 5. Net bending angle of reference thin sheet specimens subject to ns laser pulses irradiation at $d=L / 3$ as a function of laser pulse energy.

\subsection{Analysis of the influence of laser spot position on the net bending angle}

In this point, the analysis of the influence of the laser incident position (laser spot centre position) on the final specimens bending is performed.
In figure 6 the deformed profiles of the clamped sheet subject to an equal-energy laser pulse $(0,048 \mathrm{~J})$ applied at different distances from the clamping base are displayed.

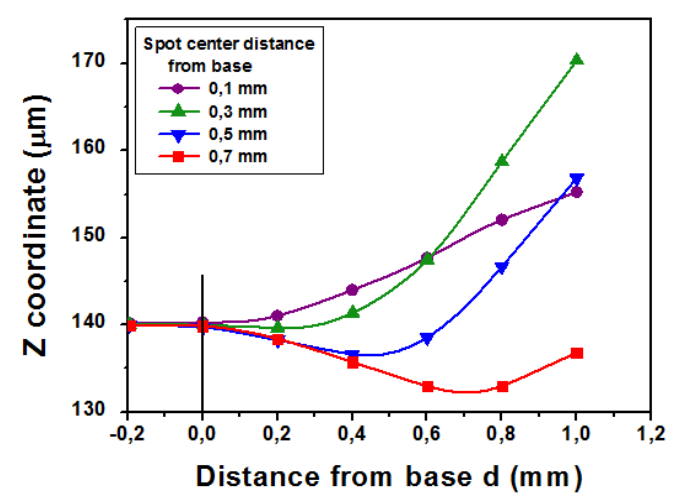

Fig. 6. Numerical deformation curves of reference thin sheet specimens subject to $0.048 \mathrm{~J}, 10 \mathrm{~ns}$ laser pulses irradiation at different distances from the pinned end.

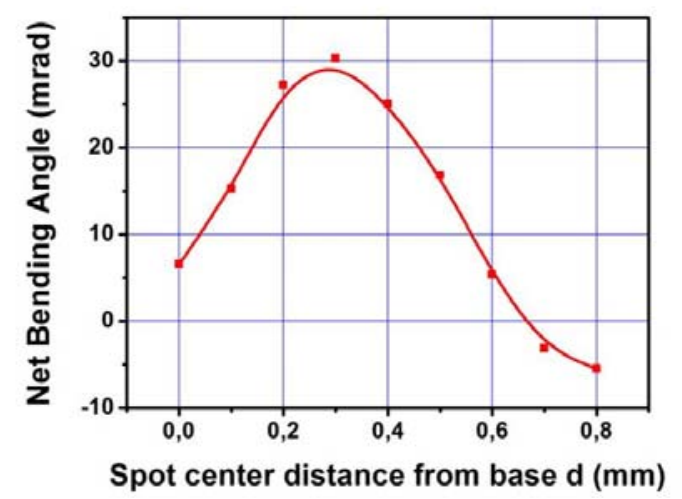

Fig. 7. Net bending angle of reference thin sheet specimens subject to $0.048 \mathrm{~J}, 10 \mathrm{~ns}$ laser pulses irradiation as a function of spot center distance from base, $\mathrm{d}$.

The study of the net bending angle of the metal sheet as a function of the laser spot position $d$ (see figure 7) shows that, as a consequence of the combination of the two main deformation effects, a maximum is obtained when the laser spot is applied at a given distance from the pinned end (about $d=L / 3$ for this process parameter).

The residual stress distribution produced by shock waves is the same in all these cases, but when the laser is near the bulk material the bending angle is reduced because it has to deform more material while the bending moment increases linearly with laser spot position.

5. Preparation of samples and Laser Shock Microforming experimental setup

The laser micromachining workstation available at the UPM Laser Centre is a multi-axis system (6 axis) integrating two UV laser sources in the nanosecond temporal regime: a Nd: $\mathrm{YVO}_{4}$ (Spectra Physics HIPPO) emitting pulsed UV radiation at $355 \mathrm{~nm}$ for focal point processing and an excimer laser (ATL LaserTechnik SP300i) emitting pulsed UV radiation at $248 \mathrm{~nm}(\mathrm{KrF})$ for mask projection processing. 
The system has been conceived as a flexible fabrication tool for developing complex laser processes in the micrometer range [10]. With the aid of the same machine it is possible to process either polymers or metallic materials. This system allows the fabrication of specimens for microforming studies with very low heat affection.

The specimens presented in this paper are Stainless Steel AISI 304 (Fe 72\%, Cr 18\%, Ni 10\%) and were machined in sets containing several stripes from a larger metal sheet. Figure 8 shows a microphotograph of the set and a detail of an individual stripe. For the conducted laser forming experiments, samples of $200 \mu \mathrm{m}$ and $300 \mu \mathrm{m}$ width were used.

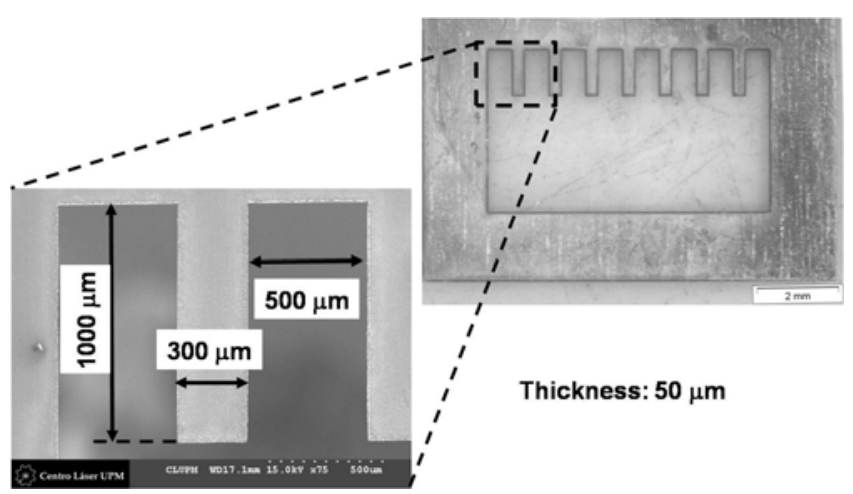

Fig. 8. Photograph of beam set and SEM photograph of individual beam with dimensions.

The geometry measurements reported in this work were made using a Confocal Laser Scanning Microscopy (Leica ICM 1000, $\lambda=635 \mathrm{~nm}$ ).

The geometry of every sample is measured to control deformations induced in the sample fabrication. In figure 9 a confocal microscopy image of the sample is presented were it can be seen that no important deformation has been induced.

The practical irradiation system used for the experiments is photographically shown in figure 10 .

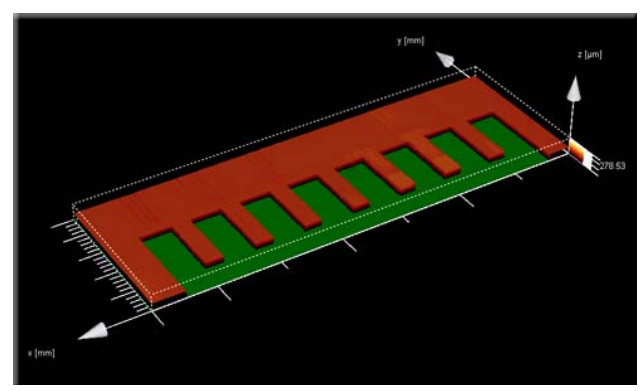

Fig.9. Confocal microscopy image of sample.

Each specimen was fixed on a holder by means of a computer controlled stage. The laser pulse is conducted to the interaction area by means of a reflecting mirror and a focussing lens (biconvex with a focal length of $203.5 \mathrm{~mm}$ ). In order to obtain a smaller spot size and to reduce the applied pulse energy, a protective mask is placed before the focussing lens.

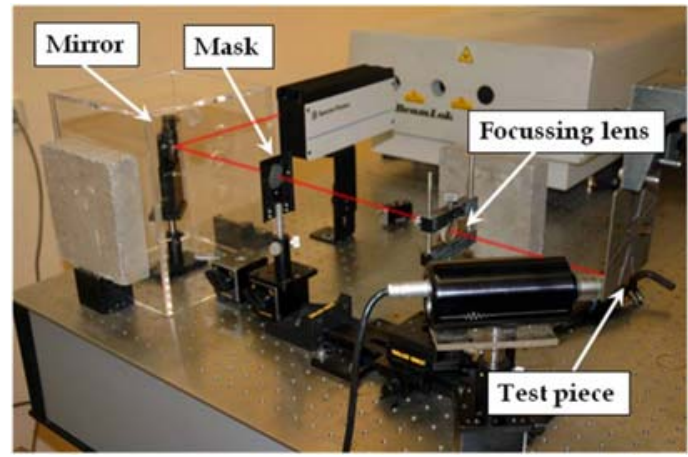

Fig. 10. Laser shock microforming experimental setup used at Centro Laser UPM.

\section{Experimental results}

The described experimental setup model has been applied to study the effect of the laser spot position and number of laser pulses on the net metal sheets bending angle. Table I shows the working conditions common to both sets of experiments.

Table I. Experimental Laser Shock Microforming Parameters

\begin{tabular}{|c|c|}
\hline Nd:YAG Laser Wavelength [nm] & 1064 \\
\hline Energy per pulse [J] & 1.651 \\
\hline Laser Pulse length FWHM [ns] & 9 \\
\hline Laser Beam radius [mm] & 14 \\
\hline Confining layer & Air \\
\hline Thin Sheet Material & AISI 304 \\
\hline Thin Sheet thickness [ $\mu \mathrm{m}]$ & 50 \\
\hline
\end{tabular}

\subsection{Influence laser spot position on net bending angle}

In order to study the influence of laser spot position on the net bending angle several experiments were systematically treated at the available experimental facility. Table II shows the corresponding working conditions.

In figure 11 a SEM photograph of the geometry of the treated specimens is shown together with the representative areas of laser interaction at each particular case.

Table II.Experimental Laser Shock Microforming Parameters

\begin{tabular}{|c|c|}
\hline Mask radius $[\mu \mathrm{m}]$ & 750 \\
\hline Energy per pulse (after mask) [mJ] & 33 \\
\hline Energy per pulse (after mask and lens) [mJ] & 31 \\
\hline Spot radius [ $\mu \mathrm{m}]$ & 175 \\
\hline Spot center position, $\mathrm{d}[\mu \mathrm{m}]$ & variable \\
\hline Beam width $[\mu \mathrm{m}]$ & 200 \\
\hline
\end{tabular}

The observed experimental deformation profiles are in good agreement with the numerical model predictions, as shown in figure 13.

\subsection{Influence of number of repeated laser pulses on the net bending angle}

In order to study the influence of number of laser pulses on the net bending angle, the samples were irradiated from 1 to 25 pulses. The microforming parameters used in the experiments are show in table III. 


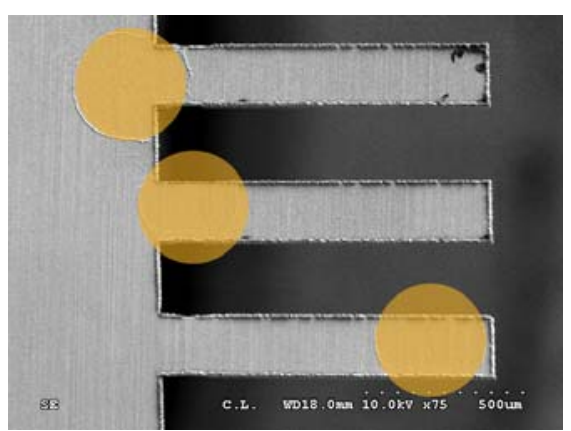

Fig. 11. Photograph of processed beams with indication of the laser incidence points in three different tests

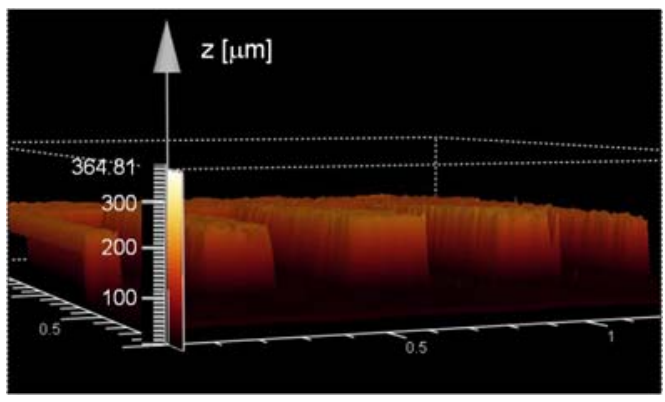

Fig. 12. Confocal microscopy image of the treated beams after processing

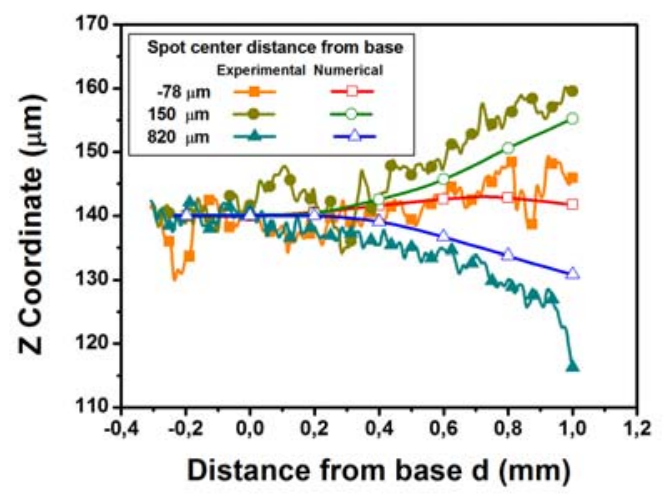

Fig. 13. Comparison of numerical vs. experimental deformation geometry for three different experimental conditions (distance from clamping base).

Table III Experimental Laser Shock Microforming Parameters for the study of the influence of number of laser pulses on net bending angle

\begin{tabular}{|c|c|}
\hline Mask radius $[\mu \mathrm{m}]$ & 750 \\
\hline Energy per pulse (after mask) $[\mathrm{mJ}]$ & 57 \\
\hline Energy per pulse (after mask and lens) $[\mathrm{mJ}]$ & 54 \\
\hline Spot radius $[\mu \mathrm{m}]$ & 150 \\
\hline Spot center position, d $[\mu \mathrm{m}]$ & 150 \\
\hline Beam width $[\mu \mathrm{m}]$ & 300 \\
\hline
\end{tabular}

Figures 14 and 15 show a SEM photograph of the beam after 25 pulses and an untreated beam from two different angles, the beam deformation being clearly observed.
The net bending angle is observed to increase with the number of laser pulses but the slope for the first pulses $(<5)$ is observed to be remarkably higher than the corresponding to a larger number of pulses $(>5)$.

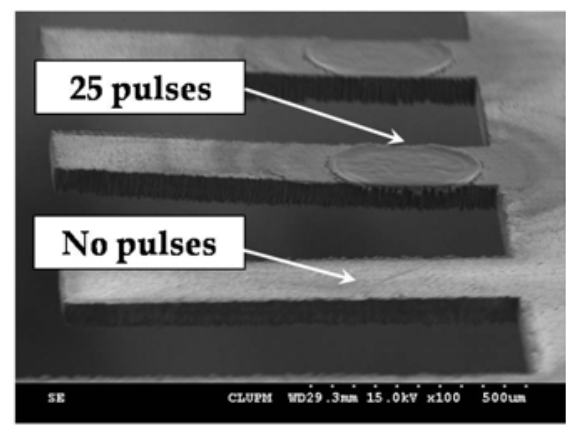

Fig. 14. Scanning electron microscope (SEM) photographs of the beam after 25 pulses and an untreated beam.

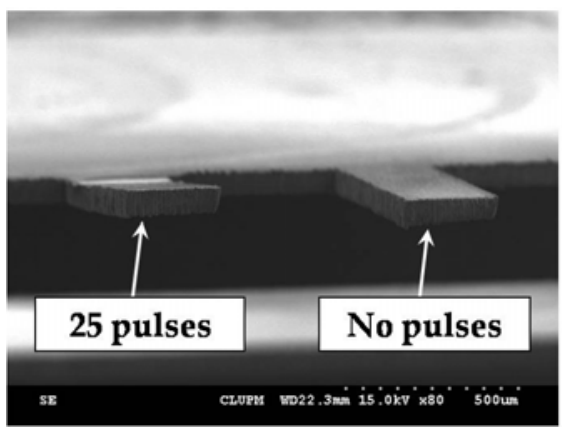

Fig. 15. Scanning electron microscope (SEM) photographs of the beam after 25 pulses and an untreated beam.

This effect, that can be observed in figure 16 and can be explained considering the combination of two reported bending mechanisms: the peak residual stress saturation after the first pulses and the increased area subjected to this residual stress distribution with every pulse.

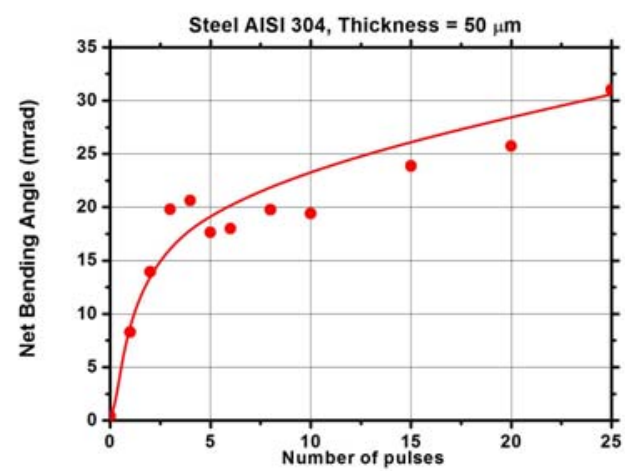

Fig. 16. Influence of a repeated number of laser pulses on the net bending angle.

\section{Discussion}

The suitability of laser microbending of thin metal strips by means of ns pulsed lasers with pulse energy in the range of a few $10 \mathrm{~mJ}$ has been experimentally demonstrated. 
From the conceptual point of view, the physics of laser shock microforming has been presented and a simulation model has been developed.

Simulations of single-end pinned targets show the presence of two opposite bending components:

1. Local bending at beam incidence position due to local plastic strain. This bending is produced in the direction of the laser beam.

2. Local bending due to bending moment in the metal stripe. This bending is produced against the direction of the laser beam.

Additionally, the simulations have shown as critical parameters:

1. Pulse energy: once a minimum (threshold) laser energy density is reached (required to produce a shock wave and plastic deformation in the material), the net bending increases with pulse energy until the bending angle due to bending moment becomes the dominant causing the saturation of the net bending angle.

2. Laser spot centre position: a maximum in global bending obtained $t$ a given position of the laser beam relative to the pinned end $(d \cong L / 3$ in the analyzed practical case). From this point on, local bending due to bending moment increases and net bending angle is reduced.

Experimental results show the influence of both parameters on the net bending angle, the observed experimental profiles being in good agreement with the numerical model predictions. Additionally the influence of a repeated number of laser pulses on the net bending angle has been characterized, the corresponding saturation effect from about $n>5$ pulses having been observed.

As a final comment, and provided that the laser forming possibilities of short pulse lasers have not only proved but also adequately characterized, according to the authors' experience, the use of ns laser pulses is considered to provide a really suitable parameter matching for the laser bending of an important range of micro mechanical systems components.

\section{Acknowledgments}

Work partly supported by Spanish MEC Projects PSE020400-2006-1, CIT0205002005-11.

\section{References}

[1] F. Vollertsen, Z. Hu, H. Schulze Niehoff and C. Theiler, Journal of Materials Processing Technology, 151 (2004) 70

[2] M. Dirscherl, G. Esser and M. Schmidt, J. Laser Micro/Nanoengineering, 1, (2006) 50

[3] H.W. Jeong, S. Hata and A. Shimokohbe, Journal of Microelectromechanical Systems, 12 (2003) 42

[4] W. Zhang, Y. L. Yao, I. C. Noyan, ASME Transactions of the ASME, Vol. 126 (2004) 10

[5] J.L. Ocaña, M. Morales, C. Molpeceres, O. García, J.A. Porro and J.J. García-Ballesteros, Appl. Surf. Sci., 254 (2007) 997
[6] J.L. Ocaña, C. Molpeceres, M. Morales and A. GarcíaBeltrán, in C.R. Phipps, M. Niino (Eds), High-Power Laser Ablation II SPIE Proceedings , Vol. 3885, 2000, p. 252.

[7] J.L. Ocaña, M. Morales, C. Molpeceres and J. Torres, Appl. Surf. Sci., 238 (2004) 242

[8] G.R. Johnson, W.H. Cook in Proceedings of the Seventh International Symposium on Ballistics, The Netherlands, The Hague, 1983, p. 541.

[9] J.L. Ocaña, M. Morales, C. Molpeceres, J.A. Porro and A. García-Beltrán, Materials Science Forum 539-543 (2007) 1116

[10]C. Molpeceres, S. Lauzurica, J.J. García-Ballesteros, M. Morales and J.L. Ocaña, Microelectronic Engineering 84 (2007) 1337

(Received: July 1, 2008, Accepted: March 11, 2009) 